\title{
Rentabilidad financiera y liquidez corriente de las empresas del sec- tor industrial que cotizan en la Bolsa de Valores de Lima, 2011-2014 Lima, Perú
}

\author{
Financial profitability and current liquidity of the companies of the \\ industrial sector that quote in the Stock exchange of Lima, 2011- \\ 2014 Lima, Peru
}
Ronald Roy Contreras Unchupaico ${ }^{11}$; Francis David Palacios Ponce de León ${ }^{1}$

1Escuela Profesional de Contabilidad, Facultad de Ciencias Empresariales, Universidad Peruana Unión

\section{INFORMACIÓN DEL ARTÍCULO}

Historia del artículo

Recibido: 13 de agosto 2016

Aceptado: 25 de octubre del 2016

Palabras clave:

Rentabilidad financiera, liquidez corriente.

\begin{abstract}
Resumen
El objetivo de esta investigación fue determinar la relación entre rentabilidad financiera y liquidez corriente de las empresas del sector industrial que cotizan en la Bolsa de Valores de Lima, periodos, 2011-2014. Es un estudio correlacional no experimental de diseño retrospectivo. La muestra poblacional está compuesta por 29 empresas del sector industrial. El análisis descriptivo tuvo los siguientes resultados: 1) el sector ha experimentado una tendencia de disminución en cuanto al nivel de rentabilidad financiera, de un nivel alto (48\%) en el 2013, hacia un nivel bajo (41\%) en el 2014; 2 ) la liquidez corriente también experimenta un caída: en el 2011 alcanzó un nivel alto (34\%) y en el 2014 un nivel bajo (45\%), 3) actualmente, las empresas se encuentran en un nivel medio de rentabilidad financiera y liquidez corriente. Según la contrastación de hipótesis se obtuvo un coeficiente de correlación de $r=-.218$ que indica la existencia de una correlación negativa inversa con un $p$ valor $=.019$. Para el 2011 se obtuvo una correlación negativa inversa $(r=-.432)$ es significativa $(p=.019)$, sin embargo, en los años 2012 ( $r=-.289, p=.128), 2013$ $(r=-.186, p=.333)$ y $2014(r=-.203, p=.291)$ existe también correlación negativa inversa pero no significativa. Se concluye que durante el periodo de estudio, las empresas experimentan una tendencia a mantener un nivel medio de rentabilidad y liquidez; sin embargo, el último año obtienen resultados de nivel bajo en ambas variables. Se concluye, principalmente que cuando las empresas experimentan mayores niveles de rentabilidad, los niveles de liquidez son menores. Sin embargo, durante los años 2012 al 2014 no siempre a mayor rentabilidad las empresas obtienen menores niveles de liquidez.
\end{abstract}

\section{Abstrac}

The aim of this investigation is to determine the relation between financial profitability and current liquidity of the companies of the industrial sector that quote in the Stock exchange of Lima, periods, 2011-2014. It is a study correlacional not experimentally of retrospective design. The population sample is composed by $29 \mathrm{com}-$ panies of the industrial sector. The descriptive analysis had the following results: 1) the sector has experienced a trend of decrease as 
for the level of financial profitability, of a high level (48 \%) in 2013, towards a low level $(41 \%)$ in $2014 ; 2$ ) the current liquidity also experiences a caída: in 2011 it reached a high level (34\%) and in 2014 a low level $(45 \%), 3)$ nowadays the empresa are in an average level of financial profitability and current liquidity. According to the contrastación of hypothesis a coefficient of correlation obtained of $r=-.218$ that indicates the existence of a negative inverse correlation with one $p$ value $=.019$. For 2011 a correlation was obtained negative inverse $(r=-.432)$ it is significant $(p=.019)$, nevertheless in the year $2012(r=-.289, p=.128), 2013(r=-.186, p=.333)$ and 2014 $(r=-.203, p=.291)$ exists also correlation negative inverse but not significant. One concludes that during the period of study, the companies experience a trend to support an average level of profitability and liquidity, nevertheless last year they obtain results of low level in both variables. One concludes, principally that when the companies experience major levels of profitability, the levels of liquidity are minor. Nevertheless during the year 2012 to 2014 not always to major profitability the companies minors obtain levels of liquidity.

\section{Introducción}

Según Gajardo y Saldivia (2013), la información sobre indicadores financieros por sectores, son de gran utilidad en términos de rendimiento y competitividad, de igual forma para la gestión y toma de decisiones en la empresa.

La gerencia suele tomar decisiones importantes acorde a cada situación de la empresa. En ese sentido, cuando se analiza el marco de liquidez corriente y rentabilidad financiera, existe escasa información de los criterios, estándares y patrones que guían para decidir sobre qué aspecto es conveniente priorizar. ¿Será ideal enfocarse netamente en generar liquidez corriente? o ¿el escenario principal debe estar dirigido a obtener rentabilidad financiera?, considerando que una de las preocupaciones importantes de la empresa es cubrir sus obligaciones a corto plazo y de los accionistas, por lo tanto, se enfoca en la obtención de la mayor rentabilidad posible.

García (2014) asegura la existencia de una relación inversa entre la rentabilidad y liquidez. En referencia, alcanzar rentabilidad no representa adquirir liquidez o la disponibilidad del efectivo en el mo- mento, como resultado, se convierte en una preocupación considerable para la gerencia, cuando la junta general de accionistas decide la distribución de los dividendos obtenidos en un cierto periodo determinado.

El presente trabajo tuvo el objetivo de determinar la relación entre la rentabilidad financiera y la liquidez corriente, así también, determinar la cantidad de empresas según los niveles de rentabilidad financiera y determinar la cantidad de empresas según los niveles de liquidez corriente, en las empresas del sector industrial que negocian en la Bolsa de Valores de Lima, 2011-2014. Es un estudio correlacional restrospectivo.

\section{Revisión de la literatura}

\section{Rentabilidad financiera}

El índice de rentabilidad representa un mensaje. Es directo y de suma importancia para los inversionistas y accionistas, que indica la eficacia, eficiencia y la productividad a través de las utilidades obtenidas de las ventas a partir del manejo adecuado de los recursos, por parte de la gerencia de la empresa. Además, la rentabilidad evalúa la capacidad y efectividad generada por 
las personas demostrada por las utilidades generadas y utilización de las inversiones (Saucedo y Oyola, 2014).

De igual forma, Pascual (2014) definió a la rentabilidad como la capacidad de un bien para producir beneficios y la medida que proporciona al compararse cuantitativamente con la inversión que lo originó.

Según Luengo, Antón, Sánchez, y Vela (2005), la rentabilidad es un elemento que se aplica a toda acción económica en la que se movilizan unos medios, materiales, humanos y financieros con la finalidad de obtener algunos resultados.

Toda empresa desea extender sus utilidades, para ello, crean estrategias para el cumplimiento de las metas propuestas. Es aquí donde el socio, tiene un papel importante; ellos invierten dinero y como consecuencia de esto, desearán obtener rentabilidad en su mayor proporción posible.

La rentabilidad económica, traducido en idioma original inglés "Return on investment" (ROI), representa la relación medida en porcentaje, entre el beneficio antes de las cargas financieras e impuestos sobre el beneficio y el volumen medio en el periodo considerado de los activos o inversión que han sido dedicados a obtener el anterior beneficio (Pascual, 2014).

La rentabilidad financiera proviene del idioma inglés: "Return on Assets" (ROA). Según Rodríguez (2013), es una medida referida a un determinado periodo de tiempo del rendimiento obtenido por los capitales propios, generalmente con independencia de la distribución del resultado. La rentabilidad financiera puede considerarse así una medida de rentabilidad más apropiada para los accionistas o propietarios que la ROI, y de ahí que sea el indicador de rentabilidad para los di- rectivos que buscan maximizar en interés de los propietarios.

\section{Liquidez corriente}

Baena Tovar (2008) definió a la liquidez corriente como la capacidad de una entidad financiera para obtener en todo momento el efectivo necesario para operar y hacer frente a sus obligaciones de pago en tiempo y a un costo razonable.

De igual forma, Rubio (2007), definió la liquidez como el nivel en que una empresa puede cancelar sus obligaciones corrientes a corto plazo. Permite medir la capacidad para satisfacer obligaciones a corto plazo conforme se venzan, es decir, alude a la solvencia de la posición financiera global de la empresa (Gitman, 2011, citado por Durán, 2011).

Por otro lado, Lee y Wong (2009), definió a la liquidez como la sensibilidad de los precios al nivel de los volúmenes negociados, por lo que un mercado es líquido si los activos se pueden comprar o vender sin tener cambios significativos en los precios; entonces, la liquidez corriente no es más que tener la capacidad de pago para cubrir con sus obligaciones de corto plazo, es decir, el ente tiene el nivel para transformar las existencias y el realizable en disponible para el uso inmediato de la empresa.

La liquidez corriente, según Aguilar (2013), relaciona los activos corrientes frente a los pasivos de la misma naturaleza; es decir, indica el grado de cobertura que tienen los activos de mayor liquidez sobre las obligaciones de menor vencimiento o mayor exigibilidad, conocido también como razón circulante, liquidez corriente y tiene específicas características: disponible, realizable y existencia. El disponible está compuesto por las partidas representativas de aquellos bienes 
que pueden ser utilizados para pagar las deudas a su vencimiento, estas son efectivo en caja, efectivo en banco e inversiones temporales (Rodríguez, 2013)

El realizable reúne aquellos bienes y derechos que habrán de convertirse en disponibles, dentro de estos se encuentran las cuentas por cobrar y los pagos anticipados (Rodríguez, 2013). Las existencias recogen el valor de los inventarios que posee la empresa y que son de su propiedad, incluyendo inventarios tanto, de materias primas en proceso y terminados, como de materiales" (Rodríguez, 2013). En ese sentido, se puede afirmar que el disponible está conformado por el dinero que posee la empresa ya sea en caja o en una institución financiera, ya sea nacional o internacional. A su vez el realizable está representado por las cuentas por cobrar que tiene la empresa pronto a convertirse en disponibles, generalmente la empresa vende su mercadería al crédito y genera una deuda en el cliente. Finalmente, las existencias son aquellas que se encuentran en el almacén de la empresa, ya sea por la compra extensa de mercadería o en su defecto porque no se logró vender lo planificado. De aquí podemos recordar el término "stock" cuando el ente compra, mas no genera ventas de lo invertido.

La falta de liquidez significa muchas veces la imposibilidad de aprovechar ventajas comerciales como descuentos por prontos pagos o posibles oportunidades de negociación con los proveedores y clientes; en otras palabras, la falta de liquidez implica libertad de elección y de movimientos por parte de la gerencia (Castillo, 2012).

En sentido contrario a lo citado en el párrafo anterior podemos mencionar que los excesos de liquidez se refieren a las causas posibles que generan el exceso de dinero en la empresa; el hecho de que en un periodo determinado del año se obtenga más ingresos o que se tenga planeado proyectos futuros y por ende no se invierta el dinero presente.

Ninguno de las dos circunstancias es favorable para el ente, se debe tener un equilibrio, el dinero no puede estar estacionado, ni tampoco se puede dar demasiadas facilidades financieras al cliente a tal punto de no contar con liquidez.

\section{Materiales y métodos}

El enfoque de esta investigación se basa en el análisis descriptivo correlacional, porque mediante el coeficiente de correlación se podrá concluir la relación que tiene la rentabilidad financiera y la liquidez corriente, y es cuantitativo porque mide las variables de estudio y aplica el análisis estadístico.

La presente es una investigación no experimental de diseño retrospectivo, puesto que este estudio se realiza con la información de años anteriores publicados en la Bolsa de Valores de Lima, sin la manipulación ni cambios de datos recolectados.

Los datos pertenecen a 29 empresas del sector industrial, publicados en la página web de la Bolsa de Valores de Lima 2011-2014. Los datos fueron analizados mediante estadísticos descriptivos para determinar el comportamiento de las variables rentabilidad financiera y liquidez corriente. También se usó estadísticos de análisis inferencial $r$ de Pearson para establecer la correlación entre la variables y dimensiones.

\section{Resultados}

Los resultados preliminares consisten en el análisis descriptivo de la cantidad 
de empresas industriales según nivel de rentabilidad financiera, y nivel de liquidez corriente. Para la prueba de hipótesis se utilzó estadístico de correlación entre las variables de estudio.

\section{Análisis descriptivo}

Tabla 1

Cantidad de empresas industriales según nivel de rentabilidad financiera de las empresas que negocian en la Bolsa de Valores de Lima, periodo 2011-2014

\begin{tabular}{ccc}
\hline Nivel & N & $\%$ \\
\hline Bajo & 35 & $30 \%$ \\
Medio & 47 & $41 \%$ \\
Alto & 34 & $29 \%$ \\
\hline Total & 116 & $100 \%$ \\
\hline
\end{tabular}

En la Tabla 1, según la distribución, la mayoría de empresas (41\%) tiene un nivel medio de rentabilidad financiera durante el periodo 211-214.

Tabla 2

Cantidad de empresas industriales según nivel de rentabilidad financiera comparativa por años de las empresas que cotizan en la Bolsa de Valores de Lima en los periodos 2011-2014

\begin{tabular}{ccccc}
\hline & \multicolumn{4}{c}{$\%$} \\
Nivel & 2011 & 2012 & 2013 & 2014 \\
& & & & \\
\hline Bajo & $17 \%$ & $21 \%$ & $41 \%$ & $41 \%$ \\
Alto & $41 \%$ & $31 \%$ & $28 \%$ & $17 \%$ \\
\hline Total & $100 \%$ & $100 \%$ & $100 \%$ & $100 \%$ \\
\hline
\end{tabular}

La Tabla 2 muestra la tendencia del nivel de rentabilidad en el periodo 2112014. Durante los años 2011 y 2012, la rentabilidad osiló entre los niveles alto y medio, mientras que la mayoría de las empresas, en los años 2013 (41\%) y 2014
(41\%), experimentaron una tendencia hacia un nivel bajo de rentabilidad

Tabla 3

Cantidad de empresas industriales según nivel de liquidez corrien te de las empresas que cotizan en la Bolsa de Valores de Lima en los periodos 2011-2014

\begin{tabular}{ccc}
\hline Nivel & $\mathrm{N}$ & $\%$ \\
\hline Bajo & 36 & $31 \%$ \\
Medio & 45 & $39 \%$ \\
Alto & 35 & $30 \%$ \\
\hline Total & 116 & $100 \%$ \\
\hline
\end{tabular}

En la Tabla 3, según la distribución, la mayoría de empresas representadas por el $39 \%$ tiene un nivel medio de liquidez corriente durante el period 211-214.

Tabla 4

Cantidad de empresas industriales según nivel de liquidez corriente comparativo por años de las empresas que negocian en la Bolsa de Valores de Lima en los periodos 2011-2014

\section{$\%$}

\begin{tabular}{lcccc} 
Nivel & 2011 & 2012 & 2013 & 2014 \\
\hline Bajo & $21 \%$ & $31 \%$ & $28 \%$ & $45 \%$ \\
Medio & $48 \%$ & $34 \%$ & $45 \%$ & $28 \%$ \\
Alto & $31 \%$ & $34 \%$ & $28 \%$ & $28 \%$ \\
\hline Total & $100 \%$ & $100 \%$ & $100 \%$ & $100 \%$ \\
\hline
\end{tabular}

La Tabla 4 muestra la tendencia del nivel de liquidez en el periodo 211-2014. Entre los años 2011 y 2013, la mayoría de las empresas tuvieron un nivel medio de liquidez corriente: $48 \%$, 34\% y $45 \%$, respectivamente, sin embargo, en el 2014 
se registra que la mayoría (45\%) de las empresas exprimentaron un nivel bajo de liquidez.

\section{Contrastación de hipótesis}

Hipótesis general

- Hipótesis nula (Ho): No existe correlación significativa entre la rentabilidad financiera y la liquidez corriente.

- Hipótesis alterna $\left(\mathrm{H}_{1}\right)$ : Existe correlación significativa entre la rentabilidad financiera y la liquidez corriente.

Regla de decisión

Si p $>0.05$, se acepta la hipótesis nula.

Si $p<0.05$, se rechaza la hipótesis nula.

Tabla 5

Análisis de correlación de rentabilidad financiera y liquidez corriente en los períodos 2011-2014

\begin{tabular}{lcccc}
\hline & \multicolumn{3}{c}{ Liquidez corriente } \\
& $\mathrm{r}$ & $\mathrm{P}$ & $\mathrm{N}$ \\
\hline Rentabilidad financiera & -.218 & .019 & 29 \\
\hline
\end{tabular}

Se observa en la Tabla 5, el coeficiente de correlación según Pearson $r=$ -.218 que indica la existencia de una correlación negativa inversa con un $p$ valor $=.019$, señalando una correlación significativa. Es decir, que a mayores niveles de rentabilidad financiera, los niveles de liquidez corriente son menores en las empresas analizadas que publican información financiera en la BVL en los períodos 2011-2014, esto también se aplica viceversa.

A continuación se presentan los resultados de correlación entre las variables rentabilidad y liquidez corriente por cada año
Tabla 6

Análisis de correlación entre rentabilidad financiera y liquidez corriente en el período 2011

Liquidez corriente

\begin{tabular}{cccc} 
& r & P & N \\
\hline Rentabilidad financiera & -.432 & .019 & 29 \\
\hline
\end{tabular}

Se observa en la Tabla 6, el coeficiente de correlación según Pearson $r=-.432$ que indica la existencia de una correlación negativa inversa con $p$ valor $=.019$, señalando una correlación significativa. Es decir, que a mayores niveles de rentabilidad financiera, los niveles de liquidez corriente serán menores en las empresas analizadas en el 2011, esto también se aplica viceversa.

Tabla 7

Análisis de correlación entre rentabilidad financiera y liquidez corriente en el período 2012

Liquidez corriente

\begin{tabular}{cccc} 
& $\mathrm{r}$ & $\mathrm{P}$ & $\mathrm{N}$ \\
\hline Rentabilidad financiera & -.289 & .128 & 29
\end{tabular}

Se observa en la Tabla 7, el coeficiente de correlación según Pearson $r=-.289$ que indica la existencia de una correlación negativa inversa con un $\mathrm{p}$ valor $=$ .128, señalando una correlación no significativa. Es decir, que a mayores niveles de rentabilidad financiera, no siempre los niveles de liquidez corriente serán menores en las empresas analizadas en el 2012.

Tabla 8

Análisis de correlación entre rentabilidad financiera y liquidez corriente en el período 2013

\begin{tabular}{ccccc}
\hline & \multicolumn{3}{c}{ Liquidez corriente } \\
& $\mathrm{r}$ & $\mathrm{P}$ & $\mathrm{N}$ \\
\hline Rentabilidad Financiera & -.186 & .333 & 29 \\
\hline
\end{tabular}

Se observa en la Tabla 8, el coeficiente de correlación según Pearson $r=-.186$, que indica la existencia de una correlación negativa inversa con p valor $=.333$. Señala una correlación no significativa. 
Es decir, que a mayores niveles de rentabilidad financiera los niveles de liquidez corriente serán menores en las empresas analizadas en el 2013.

\section{Tabla 9}

Análisis de correlación entre rentabilidad financiera y liquidez corriente en el período 2014

\begin{tabular}{cccc}
\hline & \multicolumn{3}{c}{ Liquidez corriente } \\
& $r$ & $\mathrm{P}$ & $\mathrm{N}$ \\
\hline Rentabilidad financiera & -.203 & .291 & 29 \\
\hline
\end{tabular}

Se observa en la Tabla 9, el coeficiente de correlación según Pearson $r=-.203$ que indica la existencia de una correlación negativa inversa con un $\mathrm{p}$ valor $=$ .291. Señala una correlación no significativa. Es decir, que a mayores niveles de rentabilidad financiera, no siempre los niveles de liquidez corriente también serán mayores en las empresas analizadas en el año 2014.

\section{Conclusiones}

Se llega a la conclusión, mediante el análisis descriptivo, que las empresas, du- rante el periodo de estudio, experimentan una tendencia a mantener un nivel medio de rentabilidad y liquidez, sin embargo, el último año del periodo (2014), la mayoría de las empresas obtienen resultados de nivel bajo en ambas variables

De acuerdo al objetivo general, se concluye en que sí existe relación ( $r=$ -0.218; $p=0.019$ ) entre la rentabilidad financiera y la liquidez corriente, en las empresas del sector industrial que negocian en la Bolsa de Valores de Lima de los períodos 2011 - 2014.

Durante los años del 2012 al 2014 si bien es cierto existe una correlación negativa inversa, dicha correlación no es signicativa, decir que cuando las empresas estudiadas experimentan mayor rentabilidad, no siempre se obtienen resultados de liquidez corriente desvavorable. 


\section{Referencias}

Aguilar Pinedo, V. (2013). Gestión de cuentas por cobrar y su incidencia en la liquidez de la empresa contratista corporación petrolera S.A.C. - año 2012. Tesis de licenciatura de Contador Público. Universidad San Martín de Porres.

Baena Tovar, N. (2008). La liquidez en los mercados financieros: repercusiones de la crisis crediticia. Comisión Nacional del Mercado de Valores.

Castillo, Y. (2012). Evaluación financiera de la liquidez en las empresas distribuidoras de productos farmacéuticos a través del ciclo de conversión de efectivo.

Durán, Y. (2011). Administración del capital de trabajo: una herramienta financiera para la gerencia de las Pyme tradicionales venezolanas. Visión Gerencial, 10(1).

Gajardo Bahamonde, T., y Saldivia Díaz, V. (2013). Análisis financiero del subsector de alimentos, bebidas y tabaco de Chile, a través de indicadores financieros 2012. Igarss 2014. Universidad Austral de Chile.

García Restrepo, L. (2014). Liquidez y rentabilidad como factor determinante en el éxito de las empresas, Universidad de San Buenaventura, Cartagena. 1-15.

Gitman, L. J., y Zutter, C. J. (2011). Principles of Managerial Finance (13th Edition). Prentice Hall.
Lee, J. K. Y., y Wong, A. Y. T. (2012). Impact of financial liberalisation on stock market liquidity: experience of China. Journal of Chinese Economic and Foreign Trade Studies, 5(1), 4-19.

Luengo, P., Antón, M, Sánchez, J., y Vela, J. (2005). Evaluación de los factores determinantes de la rentabilidad y solvencia de la empresa murciana. Servicio de Estudios, Cuadernos de Investigación. Colegio de Economista Región Murcia, (3).

Pascual, M. (2014). Conceptos financieros: liquidez, solvencia y rentabilidad. Recuperado de http://webs. ono.com/martinpascual/pv70601_ tresconceptos.pdf

Rodríguez Castro, D. (2013). Administración del capital de trabajo y su influencia en la rentabilidad en la Empresa Consorcio Roga S.A.C. Trujillo 2011-2012. Tesis de Licenciatura. Universidad Privada Antenor Orrego

Rubio Domínguez, P. (2007). Manual de análisis financiero. Retrieved from www.eumed.net/libros/2007a/255/

Saucedo, L., y Oyola, E. (2014). La administración del capital de trabajo y su influencia en la rentabilidad del club deportivo ABC SA. Tesis de Licencitura de Contador Público. Universidad Católica Santo Toribio de Mogrovejo 